\title{
Graphene Photo Detector with Integrated Waveguide Biochemical Sensors
}

\author{
Taehyun Hwang ${ }^{1}$, Jang Ah Kim ${ }^{3}$, Atul Kulkarni ${ }^{1,2^{*}}$ and Taesung Kim ${ }^{1,3^{* *}}$ \\ ${ }^{1}$ Department of Mechanical Engineering, Sungkyunkwan University, Suwon 440-746, Korea \\ ${ }^{2}$ Department of Physics, Sungkyunkwan University, Suwon 440-746, Korea \\ ${ }^{3}$ SKKU Advanced Institute of Nano Technology (SAINT), Sungkyunkwan University, Suwon 440-746, \\ Korea \\ *atulnano@skku.edu, ${ }^{* *} t k i m @ s k k u . e d u$
}

\begin{abstract}
In this study, we demonstrate the fabrication of graphene integrated waveguide device with graphene as a photo detector for bio-chemical sensor applications. The sensor principle relies on local evanescent wave intensity variation due to variation in analyte refractive index. For this purpose the graphene is transferred on to the field-effect transistor device and overlay of Polydimethylsiloxane (PDMS) is used as a waveguide. A $2 \mu \mathrm{l}$ drop of glucose is placed on the PDMS and the change in the photocurrent is monitored. We observed that due to change in the refractive index of the glucose the local evanescent field has changed and hence the photoconductance of the graphene. We belive that this presented technology can be used for various other bio-chemicals.
\end{abstract}

Key words: graphene, waveguide, bio-chemical sensor, filed-effect transistor, glucose

\section{Introduction}

With changing lifestyle and limitation in traditional method of detection of diseases, drug discovery, proteomics, and environmental detection of biological agents is an extremely significant problem [1]. Traditional methods used for detecting bioactivity like DNA hybridization are too slow and labor intensive. Traditional biosensors find restricted use due to limiting detecting range. They require preprocessing resulting in economic and efficiency loss. Bulk size and complicated electronic methods employed to transduce the biological functions occurring operations of these biosensors have led to new invention for detection of bioactivity. Few methods used for monitoring bioactivities include difference at the electronic supports for electrical transduction, such as current, potential changes, piezoelectric transduction, field-effect transistor (FET) transduction, photo electrochemical transduction [2] and others. To overcome these difficulties occurring in these methods new biosensors are developed to improve detection of bioactivity. New methods make use of metallic and semiconducting nanomaterials for fabrication of biosensors. Graphene is currently the subject of intense research for condensed matter physicist and electronic engineers due to its exceptional properties. Graphene also holds great promise for novel photonic devices as a photo detector [3]. Such graphene photo detectors have been deployed to optical data link applications.

In this paper we report fabrication of graphene integrated waveguide device with graphene as a photo detector for bio-chemical sensor applications. The operating principle of this sensor is based on change in photo conductance of graphene depending on refractive index (RI) of the bio-chemicals under evaluation. In this sensor graphene serves as a photo detector. This gives us the advantage of different RI for analysis of different bio-chemical activities on a single chip. Objective of our work is to fabricate bio-chemical sensor which has fast response and is sensitive to change in the bio-chemical activity.

\section{Experiment}

First, graphene film is synthesized by thermal chemical vapor deposition (CVD) method with methane and hydrogen gases, on the copper foil as a catalyst substrate. Meanwhile, FET device composed of gold/silicon oxide/silicon is used as the sensor substrate. The synthesized graphene film is transferred on the FET device. The sensor comprising graphene coated sensing region is shown in Fig. 1 (a). 
Figure 1 (b) describes the schematic of experimental setup employed in this study. As light source $(\lambda=532 \mathrm{~nm})$. I-V characteristics are observed by semiconductor parameter analyzer (SPA, 4200 SCS, and Keithley) and the output light is collected by spectrometer to measure the change in intensity of light. The various concentrations of glucose used are 60 , $70,80,90,100$ and $110 \mathrm{mg} / \mathrm{dl}$.

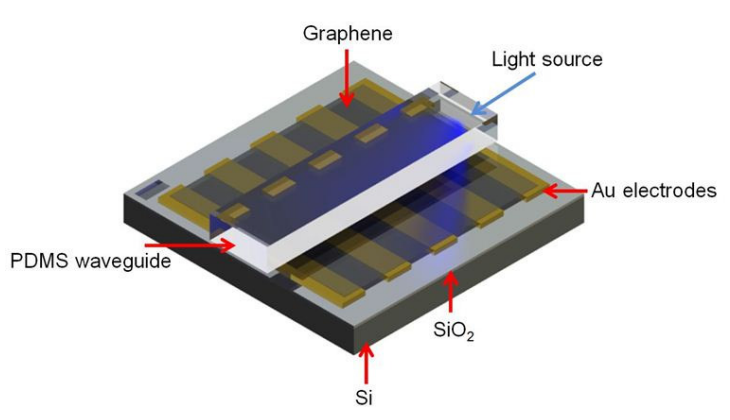

(a)

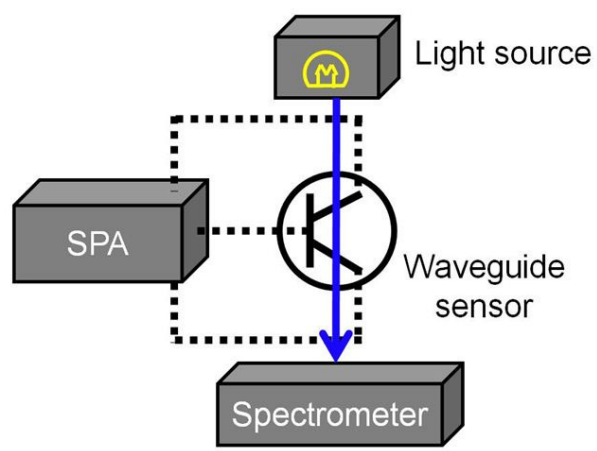

(b)

Fig. 1: Schematic of (a) waveguide FET sensor device consisting Graphene/PDMS waveguide and (b) experiment setup consisting SPA for electrical properties and spectrometer for optical properties measurments

\section{Results and Discussion}

Figure 2 and 3 shows the IV characteristics and optical intensity for used glucose solutions depending on the concentration. We observed the change current and intensity depending on the concentration of glucose. According to the relation between evanescent field and penetration depth, when the RI is increasing then penetration depth is increasing. As the penetration depth increases, the amplitude of evanescent field in the sample grows and the energy delivered to the graphene will get reduced. This affects free-electron mobility in graphene causing increase in resistance (as shown in fig. 2 inset).

Our preliminary observations on fabricated sensor device show the feasibility to use this device for sensitive bio-chemical sensor applications.

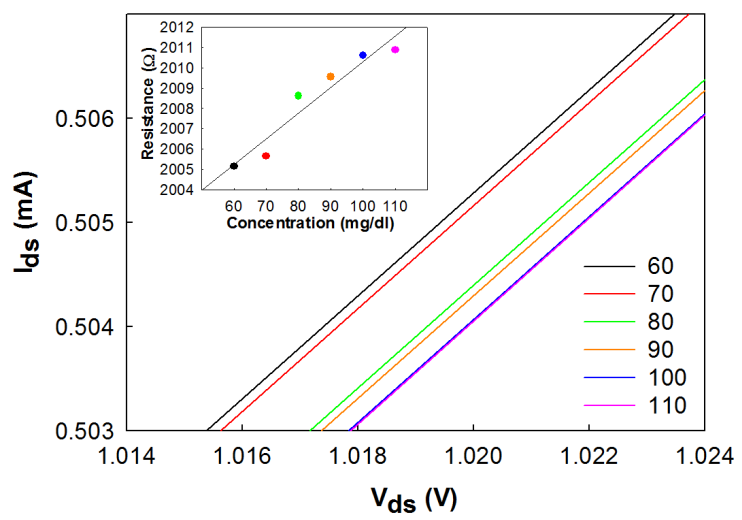

Fig. 2: I-V charactristcs for different concentrations of glucose with inset figure for the change in resistance comparisons

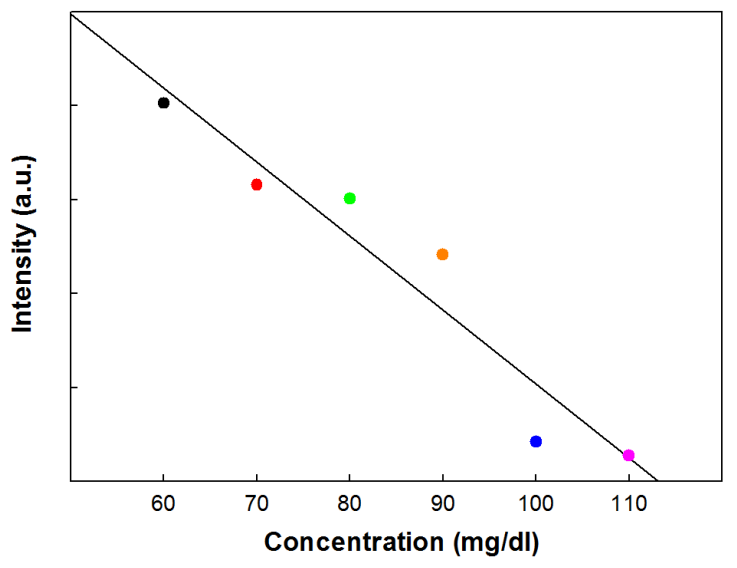

Fig. 3: Optical intensity for different concentrations of glucose

\section{Acknowledgements}

This work was partly supported by the GRRC program of Gyeonggi province [S-2011-1039007-1, Development of integrated sensor module for measurement of TVOCs and PM2.5 from vehicle emission] and Basic Science Research Program through the National Research Foundation of Korea (NRF) funded by the Ministry of Education, Science and Technology 


\section{References}

[1] Turner, A. P. F., Biosensors--Sense and Sensitivity, Science 290, 1315-1317 (2000); doi: 10.1126/science.290.5495.1315

[2] V. Pardo-Yissar, E. Katz, J. Wasserman, I. Willner, J. Am., Acetylcholine Esterase-Labeled CdS Nanoparticles on Electrodes: Photoelectrochemical Sensing of the Enzyme Inhibitors, Chemical Society 125, 622-623 (2003); doi; 10.1021/ja028922k

[3] K. F. Mak et. al., Measurement of the Optical Conductivity of Graphene, Physical Review Letter 101, 196405-196408 (2008); doi: 10.1103/PhysRevLett.101.196405 